\title{
Percepción del consumo de noticias en línea y de las prácticas relacionadas a ellas por parte de los jóvenes de la Comunidad de Madrid (España)
}

\section{Perception of online news consumption and young people related practices in the Community of Madrid (Spain)}

\section{Percepção do consumo de notícias online e práticas afins dos jovens da Comunidade de Madrid (Espanha)}

Beatriz Catalina-García, Universidad Rey Juan Carlos Fuenlabrada, Madrid, España (beatriz.catalina@urjc.es)

Antonio García-Jiménez, Universidad Rey Juan Carlos Fuenlabrada, Madrid, España (antonio.garcia@urjc.es)

Pedro Paniagua-Santamaría,Universidad Complutense de Madrid, Madrid, España (ppaniagu@ucm.es)

RESUMEN | Este artículo analiza los hábitos de consumo de noticias de los jóvenes de la Comunidad de Madrid, su percepción sobre estas y las prácticas relacionadas. Conocer su comportamiento en línea es relevante y tiene consecuencias en la actividad periodística. A partir de una encuesta estadísticamente representativa, aplicada entre junio y julio de 2019, se obtuvo el punto de vista de 533 participantes (17-24 años), estudiando la influencia de la edad y el nivel de estudios. La investigación confirma el bajo consumo de noticias por parte de los jóvenes. Además de la presencia general del consumo en línea, se corrobora el acceso incidental como característica en este sector de la población, modulado por la edad. También se profundiza en las temáticas con mayor preferencia, las vías de mayor prevalencia para consumir noticias en línea, los mecanismos empleados para compartirlas, y las prácticas relacionadas con la verificación. Finalmente, se observa la importancia de la comunicación presencial en el consumo de noticias y las diferencias existentes entre consumo y confianza en el medio de acceso a la noticia.

PALABRAS CLAVE: consumo de noticias; jóvenes; medios sociales; percepción. 
ABSTRACT / This article analyses the news consumption habits of young people in the Community of Madrid (Spain), their perception about them and the practices related to it. This is a relevant question, both for understanding the online behavior and for the effect it has on journalistic activity. Based on a statistically representative survey applied between June and July 2019, we obtained the point of view of 533 participants (17-24 years), and the influence of age and level of education is studied. The research confirms the low rate of news consumption of young people. In addition to the general presence of online consumption, incidental access is confirmed as a characteristic of this sector of the population, though it is influenced by age. It also explores the most preferred topics, the most prevalent ways of online news consumption, the procedures used to share them, and the practices related to verification. Finally, the importance of face-to-face communication in news consumption and the differences between consumption and trust in the means of accessing the news are observed.

KEYWORDS: news consumption; youth; social media; perception.

RESUMO | Este artigo analisa os hábitos de consumo de notícias dos jovens da Comunidade de Madrid, sua percepção das notícias e práticas afins. Conhecer o seu comportamento online é relevante e tem consequências para a atividade jornalística. De um inquérito estatisticamente representativo aplicado entre junho e julho de 2019, foi obtido o ponto de vista de 533 participantes (17-24 anos ) estudando a influência da idade e do nível educacional. A investigação confirma o baixo consumo de notícias por parte dos jovens. Além da presença geral do consumo online, o acesso acidental é corroborado como uma característica neste setor da população, embora modulado por idade. Também se aprofunda sobre os temas mais preferidos, as formas mais prevalecentes de consumir notícias online, os mecanismos utilizados para as compartilhar e as práticas relacionadas com a verificação. Por fim, é observada a importância da comunicação presencial no consumo de notícias e as diferenças existentes entre consumo e confiança nos meios de acesso às notícias.

PALAVRAS-CHAVE: consumo de notícias; jovens; mídias sociais; percepção. 


\section{INTRODUCCIÓN}

El consumo de noticias es uno de los factores clave para el desarrollo de los jóvenes como ciudadanos, quienes enfrentan el crecimiento exponencial de nuevas vías de acceso en línea a productos periodísticos. Recurren a blogs, aplicaciones de mensajería instantánea y, especialmente, a los medios sociales, por su facilidad de acceso y la interactividad que aportan a los usuarios. Los jóvenes no solo consumen contenidos, sino también generan informaciones y opiniones, y las comparten en un corto lapso entre amplios círculos sociales (Catalina-García et al.,2019).

La adopción natural de las nuevas tecnologías que caracteriza a los jóvenes (Yuste, 2015) se asocia con una tendencia del consumo de las noticias en el entorno digital, en detrimento de los medios convencionales (Fernández de ArroyabeOlaortua et al., 2018). En un contexto en el que los valores propios de un buen periodismo -autenticidad de las fuentes utilizadas, veracidad y objetividadson puestos en entredicho, se difumina la diferencia entre estar informado y estar bien informado.

Este trabajo analiza el consumo de noticias de los jóvenes de la comunidad de Madrid, su percepción sobre estas y las prácticas relacionadas a ellas. Nos interesa saber si la selección de noticias procede de prácticas escasamente críticas para discriminar contenidos. De ser así, este fenómeno posiblemente sería una consecuencia de cuestiones como la rapidez para el acceso o la desafección por lo institucionalizado.

\section{ESTADO DE LA CUESTIÓN}

\section{Patrones y tipos de comportamiento}

El consumo de noticias por parte de los jóvenes es un tema relevante, entre otras cuestiones, por el impacto en la configuración de la actividad periodística del futuro. También influye en la esfera política e incluso en las propias percepciones e intenciones de compra (Wackowski et al., 2019).

Por ello, es relevante considerar sus actitudes ante las noticias, su nivel de consumo y sus diferentes usos. En términos generales mantienen actitudes positivas hacia los sitios de noticias en línea (Kang, 2009), con preferencia por el acceso digital ya desde hace tiempo. No obstante, no hay acuerdo sobre su grado de consumo de información de actualidad. Aunque hay autores que apuntan que a un porcentaje relevante les gusta estar informados y ese interés parece aumentar con la edad (Costera, 2007; Casero-Ripollés, 2012), fundamentalmente a través de los medios sociales (Kim et al., 2014), otros alertan sobre la disminución en su consumo de los medios de comunicación (Drok et al., 2018). 
Esto último se puede achacar a un menor interés por cuestiones políticas o sociales, o incluso a un descenso en la cultura cívica. También se podría considerar que los jóvenes sí están comprometidos socialmente, pero no siguen a los medios de comunicación tradicionales, porque les son cada vez más irrelevantes.. En comparación con la prensa, los jóvenes señalan la rapidez y el precio como las principales razones del consumo digital (Cherian \& Jacob, 2012).

No todos los jóvenes consumen noticias. Conforme a Edgerly y sus colegas (2018a), 52\% de ellos tendería a ignorarlas. Así, una mayor oferta de información no tendría como consecuencia un mayor consumo de noticias (Arceneaux \& Johnson, 2013). Esta conducta coincide con investigaciones realizadas entre usuarios adultos (Hasebrink \& Popp, 2006; Lee \& Yang, 2014).

Entre los jóvenes que sí consumen noticias se podrían identificar dos grupos. Por un lado, los que buscan las noticias nacionales y locales más importantes, independientemente de los dispositivos. Suelen estar relativamente más interesados en la política y, de la misma forma que sus homólogos adultos (Lee \& Yang, 2014), buscan información de actualidad en todas las fuentes posibles. Este hecho está en consonancia con el descubrimiento de que se mantiene el hábito de contacto con las cabeceras tradicionales, fundamentalmente en formato en línea (Kaufhold, 2010; Hermida et al., 2012; Condeza-Dall'Orso et al., 2014; CatalinaGarcía et al., 2015).

No obstante, crece el número de jóvenes que toma en cuenta sitios web de redes sociales y agregadores de información para estar al día sobre acontecimientos y eventos. Este perfil podría desglosarse en dos subtipos: quienes se involucran activamente en buscar contenidos en las redes y los que se conectan con la información de forma incidental, debido a comentarios de amigos o compañeros y al resultado de algoritmos que calculan qué noticias están en sus preferencias. Ambos tipos de usuarios han sido considerados como buscadores y stumblers, respectivamente (Bode, 2016; Kim et al., 2013).

En el ámbito audiovisual, Papathanassopoulos y sus colegas (2013) confirman que los más jóvenes tienden a reemplazar los canales tradicionales de noticias. Wonnenberger y Kim (2017) muestran que, en general, ven menos noticias en televisión cuando hay más canales alternativos disponibles.

\section{Consumo incidental}

En términos generales, los jóvenes no buscan deliberadamente las noticias, sino que las consumen de forma incidental, aunque hay diferencias de percepción sobre la frecuencia del consumo (Patterson, 2007; Qayyum et al., 2010; Bergström \& Jervelycke Belfrage, 2018). 
Entre los factores que influyen en su consumo de noticias, Bergström y Jervelycke Belfrage (2018) apuntan al interés informativo, a la influencia de líderes de opinión, dado que ofrecen un marco de interpretación de las noticias y a la edad. A mayor edad, menor consumo incidental. Otra circunstancia relevante es la familiar. El tipo de consumo de los padres de adolescentes entre 12 y 17 años es un factor determinante: Edgerly y sus colegas (2018b) incluso se refieren a un modelo emparejado de consumo informativo entre ambos.

El dispositivo móvil es el artefacto más empleado para consumir noticias (Boczkowski et al., 2017; Vara-Miguel, 2019). Con un consumo incidental, las noticias se convierten en un contenido más dentro de los contenidos de entretenimiento a los que acceden los jóvenes, a las que dedican poco tiempo, fenómeno común en los estudiantes universitarios (Catalina-García et al., 2019).

La intensidad en la actividad digital también puede influir en el consumo de noticias. A mayor tiempo de consumo, mayor es el acceso a las noticias y su verificación (Catalina-García et al., 2015). Por otra parte, los jóvenes consumen noticias por motivaciones normativas (entre las que destaca ser un buen ciudadano) y por intereses personales, educativos, profesionales o pasatiempos (Brites \& Kõuts-Klemm, 2018).

\section{Redes sociales y noticias}

Para entender el consumo de noticias por parte de los jóvenes, es pertinente detenerse en las relaciones entre acceso a las noticias y las redes sociales (Bergström \& Jervelycke Belfrage, 2018; Mitchelstein \& Boczkowski, 2018; Mumtaz et al., 2019). Mitchelstein y Boczkowski (2018) consideran el uso de Facebook y Twitter como uno de los factores asociados a la probabilidad de consumo incidental de información periodística. Otro factor es la edad, pues los más jóvenes tienen más probabilidad de conectarse con la información de actualidad de este modo. El creciente acceso a las noticias vía WhatsApp parece confirmarlo (Martínez Costa et al., 2019).

Los usuarios de redes sociales utilizan más fuentes de información de manera incidental, especialmente los más jóvenes con poco interés en este tipo de contenidos (Fletcher \& Nielsen, 2018). A juicio de Gangadharbatla y sus colegas (2014), los jóvenes entienden que los medios sociales favorecen el control sobre la información recibida frente a otras opciones. Acceden a las noticias solo en determinadas circunstancias, a través de medios sociales, para después consultar los medios de comunicación digitales, en muchos casos tradicionales, a los que conceden mayor credibilidad (Hilligoss \& Rieh, 2007; Flanaging \& Metzer, 2008; Johnson \& Kaye, 2014). 
Por otro lado, diversos estudios coinciden en que el grado de credibilidad que los jóvenes y adolescentes otorgan a las noticias en línea es alto (Catalina-García et al., 2015; Flanagin \& Metzer 2008; Kang, 2009).

El consumo de noticias a través de Facebook o WhatsApp tiene diversas conexiones con las relaciones sociales. Quienes presentan lazos débiles dentro de la red prestan más atención a la información de actualidad. Al compartir noticias dentro de una comunidad, el control percibido sobre la privacidad, la presencia de normas claras y límites comunitarios, y los objetivos comunicativos del grupo son factores clave (Swart et al., 2019).

\section{El caso español}

Los jóvenes españoles (15-29 años) conforman un colectivo caracterizado por vivir en el seno de familias con cambios estructurales. Presentan altas cifras de desempleo y prolongan sus estudios (Benedicto, 2017). La mediación tecnológica influye en sus prácticas sociales, desconfían de las instituciones y tienen una práctica asociativa baja. En una línea similar está el resto de los europeos del oeste: aprueban menos a los medios de comunicación que en edades superiores, aunque siguen confiando en las marcas de los periódicos (Matza et al., 2018). Además, son mucho más propensos a nombrar los medios de comunicación social (31\% según Vara-Miguel et al., 2019) y los sitios de motores de búsqueda como principales fuentes de noticias, superando a la televisión (27\%) y a la prensa en papel.

Precisamente. las redes sociales se alzan como la principal fuente de noticias entre los jóvenes españoles de 18 a 24 años, según el informe Digital News Report España (Negredo et al., 2021), a pesar de que la población en general considera que son las que más desinforman. En términos comparativos, los datos extraídos son similares en países iberoamericanos (Argentina, Brasil, Chile y México) incluidos en el informe de Newman y sus colegas (2020): los medios sociales se consolidan cada vez más como fuente de consulta, aunque la confianza en las noticias en general disminuye, incluidas las procedentes de los medios sociales, pero también de los periodísticos. Chile y España muestran una grave caída de la confianza en estas marcas profesionales.

El consumo de noticias también se asocia a episodios breves e intermitentes, como ráfagas de información, o picoteo informativo, propios de los dispositivos móviles (Martínez et al., 2019). Algunos estudios apuntan a que muchos universitarios no se informan con rigor (Soengas-Pérez et al., 2019). No obstante, los jóvenes (1834 años) conforman el grupo de edad que más compara las fuentes de noticias en la web y los que más utilizan diversas fuentes de noticias a través de Twitter o diferentes blogs (Hernández-Serrano et al., 2017). 


\section{OBJETIVOS}

Este estudio analiza el consumo de noticias por parte de los jóvenes de la Comunidad de Madrid, a partir de la edad (17 a 24 años) y del nivel de estudios. Se plantean los siguientes objetivos:

1. Comprobar los hábitos de consumo de noticias en medios, ámbitos de interés y frecuencia de acceso.

2. Establecer las acciones que realizan con las informaciones, tanto las que consultan como las que les llegan incidentalmente. Si las contrastan o no, por qué lo hacen, y si las comparten.

3. Conocer el grado de credibilidad que les inspira cada medio y cuáles son, si los hay, los elementos propios de una información que les motiva para confiar en el contenido de las noticias.

4. En términos también de percepción, se indaga sobre las motivaciones que les impulsan a informarse.

\section{METOdOLOGÍA}

Se administró un cuestionario a una muestra estadísticamente representativa de 533 jóvenes de entre 17 y 24 años de la Comunidad de Madrid entre el 17 de junio y el 4 de julio de 2019, de forma personal, en sus domicilios. La captación de la muestra y la aplicación fue encargada a una entidad especializada en técnicas sociológicas cuantitativas. La encuesta fue aprobada por el comité de ética de la investigación de la Universidad Rey Juan Carlos $^{1}$ y el estudio sigue la normativa española (Reglamento de la Unión Europea 2016/679) relativa a la protección de datos.

El error de análisis queda fijado en $\pm 4,33 \%$ para datos globales bajo el supuesto de $p=q=0,5$, con un nivel de confianza de $95 \%$. Con este fin se aplica un muestreo bietápico, estratificado por conglomerados, con selección de las unidades primarias de muestreo (municipios y secciones censales) de forma aleatoria proporcional y de las unidades últimas (individuos) por rutas aleatorias y cuotas de sexo y edad, según los datos correspondientes a la población interpolada por edad simple, para cada sexo con fecha de referencia de 1 de enero del 2019 (provisional) para la Comunidad de Madrid y facilitados por el Instituto Nacional de Estadística (2019) ${ }^{2}$. El acceso a la información se realizó por el Sistema C.A.P.I. (encuesta personal asistida con ordenador en el hogar de los encuestados). La duración media de las encuestas fue de 25 minutos.

1. Número de registro interno: ENM 53/172103201906619. Fecha de la firma: 10/04/2019

2. https://www.ine.es/ 


\begin{tabular}{c|ccc|ccc} 
& \multicolumn{3}{|c}{ Muestra } & \multicolumn{3}{c}{ Población Comunidad Madrid y } \\
muestra de peso
\end{tabular}

*Población interpolada por edad simple, para cada sexo. Fecha de referencia 1/1/2019 (provisional) para la Comunidad de Madrid (INE, 2019).

Tabla 1. Distribución de la muestra y de la población por sexo y grupos de edad Fuente: Elaboración propia con base en datos del INE de España.

Tras la recolección de los datos, se detectó una ligera desviación de las variables de sexo y edad de la muestra con respecto a las registradas en el universo de los jóvenes de la Comunidad de Madrid. Se ajustó la muestra para mantener su representatividad (tabla 1).

El nivel educativo se separó en dos franjas: nivel superior y no superior, de tal forma que el porcentaje acumulado de los primeros es de $35,4 \%$ y de $64,6 \%$ entre los de estudios superiores.

\section{Características del cuestionario}

El cuestionario, de elaboración propia, se basa en otros estudios relacionados que utilizaron esta técnica (Aramburú-Moncada \& López-Redondo, 2018; Catalina-García et al., 2015; Condeza- Dall'Orso et al., 2014), con tres ejes temáticos. Consumo de noticias: examina los medios empleados para informarse; incluye versiones tradicionales y en línea de productos periodísticos (prensa, radio y televisión) y otras fuentes digitales (redes sociales, buscadores, blogs, boletines de suscripción). Se indaga si este consumo es incidental, por transmisión en su entorno familiar o de amistad a través de alguna vía (teléfono o servicios de mensajería, por ejemplo) o directamente en el contacto cara a cara. El uso de los medios y formas de informarse se miden con una escala temporal ascendente: nunca, alguna vez al mes, una o dos veces por semana, tres o cuatro veces por semana, todos o casi todos los días. En segundo término, se examinan las temáticas clasificadas según las secciones tradicionales de prensa: política, economía, sucesos, catástrofes, medioambiente, cultura, deportes, ciencia, tecnología, curiosidades y entretenimiento, agenda de servicios y famosos/ celebrities (encuadrada mayoritariamente en la prensa rosa). 
1. Acciones que ejercen con la información: se observa la frecuencia y causas que les incitan (o no) a contrastar la información y si las comparten o comentan con su entorno. En caso positivo, cómo lo hacen, si en su entorno offline o a través de medios digitales. Se mide la frecuencia mediante una escala de Likert (nunca, rara vez, en ocasiones, con mucha frecuencia y continuamente).

2. Confianza y percepción sobre las distintas vías que generan información: para ambos aspectos, se aplica igualmente la escala de Likert. En el caso de la confianza, se mide el nivel que les inspira cada uno de los citados medios empleados. Se pregunta sobre los rasgos destacados para considerar que una noticia es creíble, según contenido, calidad del medio, inclusión de contenidos audiovisuales, autoría o fuente y publicación en dos o más medios. En cuanto a la percepción, se solicita marcar su grado de acuerdo/desacuerdo con una serie de afirmaciones para indagar por qué consultan noticias. Para explorar cuán importante consideran informarse relacionándolo con su puesta en práctica: si creen que es fundamental para tomar decisiones, si solo les interesa aquello que les afecta directamente, o si a pesar de que reconocen que es conveniente, no suelen consultar.

Los datos recopilados fueron tratados y analizados con el programa SPSS Statistics v26 y el nivel de validez estadística se establece en $c^{2}<0,05$.

\section{RESULTADOS}

\section{Consumo de noticias}

A excepción del formato convencional de la televisión (39,8\% varias veces a la semana y $34,8 \%$ todos o casi todos los días), los jóvenes apenas acceden a las noticias en medios tradicionales. El 55,8\% asegura que nunca o alguna vez al mes recurren a la radio analógica para informarse y solo 5,8\% lo hace a diario o casi siempre, mientras que $40,5 \%$ nunca accede a la prensa en papel y únicamente 5,9\% lo hace habitualmente (todos o casi todos los días).

Aunque las versiones en línea de estos medios tienen una aceptación mayor, la prensa en línea es la más frecuentada por los jóvenes: 29,5\% accede como mínimo tres o cuatro veces por semana, lo mismo para la radio digital $(20,1 \%)$ y la televisión en línea, por una cuarta parte (tabla 2).

Resulta significativo que buena parte de los encuestados no buscan las noticias: $69,6 \%$ las conoce habitualmente por la familia y $70,3 \%$, por sus amigos o compañeros a través de WhatsApp o cara a cara. Esta forma incidental de informarse se produce, al menos, tres o cuatro veces por semana. Además de la televisión convencional, esta frecuencia solo se aproxima a la consulta que $48,2 \%$ hace deliberadamente en buscadores sobre temas que les interesa (tabla 3 ). 


\begin{tabular}{ccccccc} 
& Nunca & $\begin{array}{c}\text { Alguna } \\
\text { vez al } \\
\text { mes }\end{array}$ & $\begin{array}{c}\mathbf{1 - 2} \text { veces } \\
\text { por semana }\end{array}$ & $\begin{array}{c}\text { 3-4 veces } \\
\text { por semana }\end{array}$ & $\begin{array}{c}\text { Todos o } \\
\text { casi todos } \\
\text { los días }\end{array}$ & NS/NC \\
\hline Prensa en papel & 40,5 & 23,6 & 15,9 & 11,7 & 5,9 & 2,4 \\
\hline Radio convencional & 36,9 & 18,9 & 22 & 14,7 & 5,8 & 1,6 \\
\hline TV convencional & 10,3 & 13,5 & 19 & 20,8 & 34,8 & 1,6 \\
\hline Prensa en línea & 25,1 & 21,4 & 20,7 & 18,5 & 11 & 3,3 \\
\hline Radio en línea & 39,4 & 20 & 18,3 & 12,6 & 7,5 & 2,2 \\
\hline TV en línea & 30,6 & 20,3 & 20,9 & 15,8 & 9,2 & 3,2 \\
\hline
\end{tabular}

Tabla 2. Frecuencia de consumo de medios (\%)

Fuente: Elaboración propia.

\begin{tabular}{|c|c|c|c|c|c|c|}
\hline & Nunca & $\begin{array}{l}\text { Alguna } \\
\text { vez al } \\
\text { mes }\end{array}$ & $\begin{array}{l}1-2 \text { veces } \\
\text { por semana }\end{array}$ & $\begin{array}{c}\text { 3-4 veces } \\
\text { por semana }\end{array}$ & $\begin{array}{l}\text { Todos o } \\
\text { casi todos } \\
\text { los días }\end{array}$ & NS/NC \\
\hline $\begin{array}{l}\text { Te llegan a través de } \\
\text { redes sociales }\end{array}$ & 7 & 8,7 & 17,1 & 28,4 & 38,1 & 0,8 \\
\hline $\begin{array}{c}\text { Te llegan a través de } \\
\text { algún boletín o lista } \\
\text { de distribución de } \\
\text { noticias }\end{array}$ & 33,2 & 12,8 & 19,6 & 18,1 & 12 & 4,4 \\
\hline $\begin{array}{c}\text { Te llegan noticias } \\
\text { sobre temas } \\
\text { específicos en } \\
\text { buscadores }\end{array}$ & 16,3 & 12,6 & 20,3 & 25,7 & 22,5 & 2,7 \\
\hline $\begin{array}{l}\text { Te llegan por } \\
\text { familiares, en } \\
\text { persona, por teléfono } \\
\text { o WhatsApp }\end{array}$ & 5,4 & 8,5 & 16,2 & 27 & 42,6 & 0,2 \\
\hline $\begin{array}{l}\text { Te llegan por } \\
\text { amigos, compañeros } \\
\text { o conocidos en } \\
\text { persona, por teléfono } \\
\text { o por WhatsApp }\end{array}$ & 4,2 & 7,7 & 17,3 & 24,9 & 45,5 & 0,5 \\
\hline
\end{tabular}

Tabla 3. Porcentajes de frecuencia de consumo incidental

Fuente: Elaboración propia. 


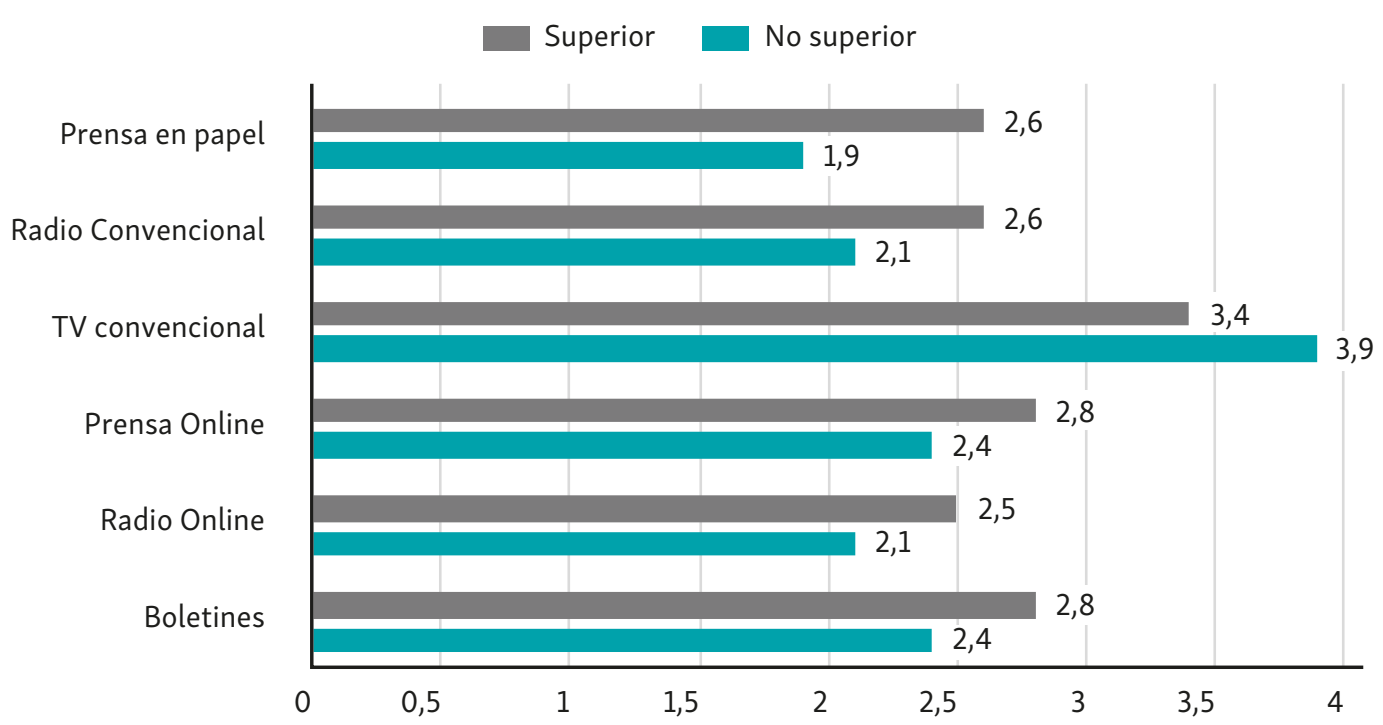

Figura 1. Medias en función del nivel educativo sobre la recurrencia a medios con diferencias significativas en función de chi cuadrado $\left(c^{2}<0,05\right)$

Fuente: Elaboración propia.

La edad marca diferencias significativas al consultar la prensa digital e impresa, y en la suscripción a boletines o listas de noticias. A mayor edad recurren más a estas formas para informarse. El 71,5\% de los encuestados entre 17 y 20 años apenas consultan las informaciones en periódicos tradicionales o revistas (nunca o alguna vez al mes), frente a 54,9\% de los del rango 21-24 años, que tampoco recurre a este medio o lo hace muy esporádicamente.

Los jóvenes con un nivel educativo superior consultan con mayor frecuencia la prensa y la radio. Los valores medios oscilan entre 3,1 de los periódicos/revistas digitales y 2,5 de la radio digital. En contraste, los jóvenes con menos estudios alcanzan su valor máximo en un 2,6 de la prensa en línea y un mínimo de 1,9 (entre nunca y alguna vez cada mes), lo que se corresponde a este mismo medio en su versión tradicional. La televisión convencional sí es una fuente de noticias algo más recurrente para las personas con estudios inferiores, cuya media de frecuencia es de 3,9 (cercano a tres/cuatro veces por semana), cinco décimas más altas que para los que tienen estudios de nivel superior. Para otras alternativas de consumo, solamente se aprecian diferencias estadísticas en las suscripciones a boletines, cuya recurrencia es algo mayor entre los grados educativos más altos (figura 1).

Curiosidades y entretenimiento, tecnología, deportes y cultura son, en ese orden, las temáticas consultadas más habitualmente a través de redes sociales y otras fuentes alojadas en Internet. En todos los casos se observa una media de consulta superior a 3 , valor que se corresponde a con bastante frecuencia. 
Los escasos y más bajos valores que alcanzan las noticias relacionadas con política y con economía ( $\delta=2,35$ sobre 5 en cada uno de los ítems) reflejan que, en general, rara vez las consultan. Esto refuerza la tradicional y ya estudiada desafección de los jóvenes por estos temas.

De hecho, la edad registra diferencias significativas para política $(\chi 2=, 000)$ y economía ( $\chi 2=, 002)$ : a medida que se hacen mayores, crece su interés por ambos. El $64,5 \%$ de los más jóvenes nunca o rara vez consulta las noticias políticas, mientras que la cifra baja a 50\% cuando la edad oscila entre los 21 y los 24 años. En relación con la economía, la diferencia es mayor: $78,1 \%$ de los jóvenes de entre 17 a 20 años no se interesa, frente a $46 \%$ de los mayores (tablas 4 y 5 ).

\section{Política}

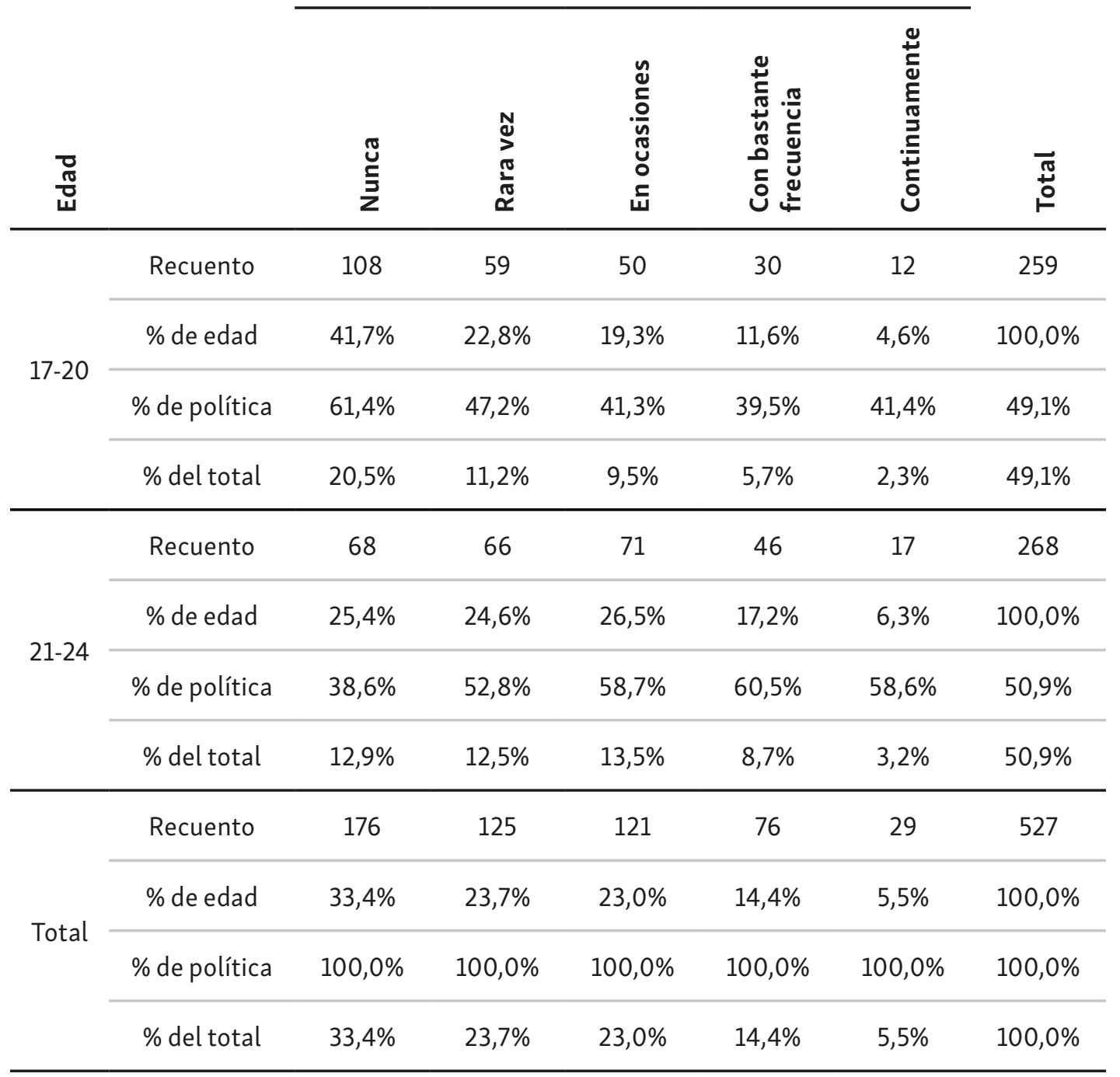

Tabla 4. Contingencia edad-consulta información política

Fuente: Elaboración propia. 
Economía

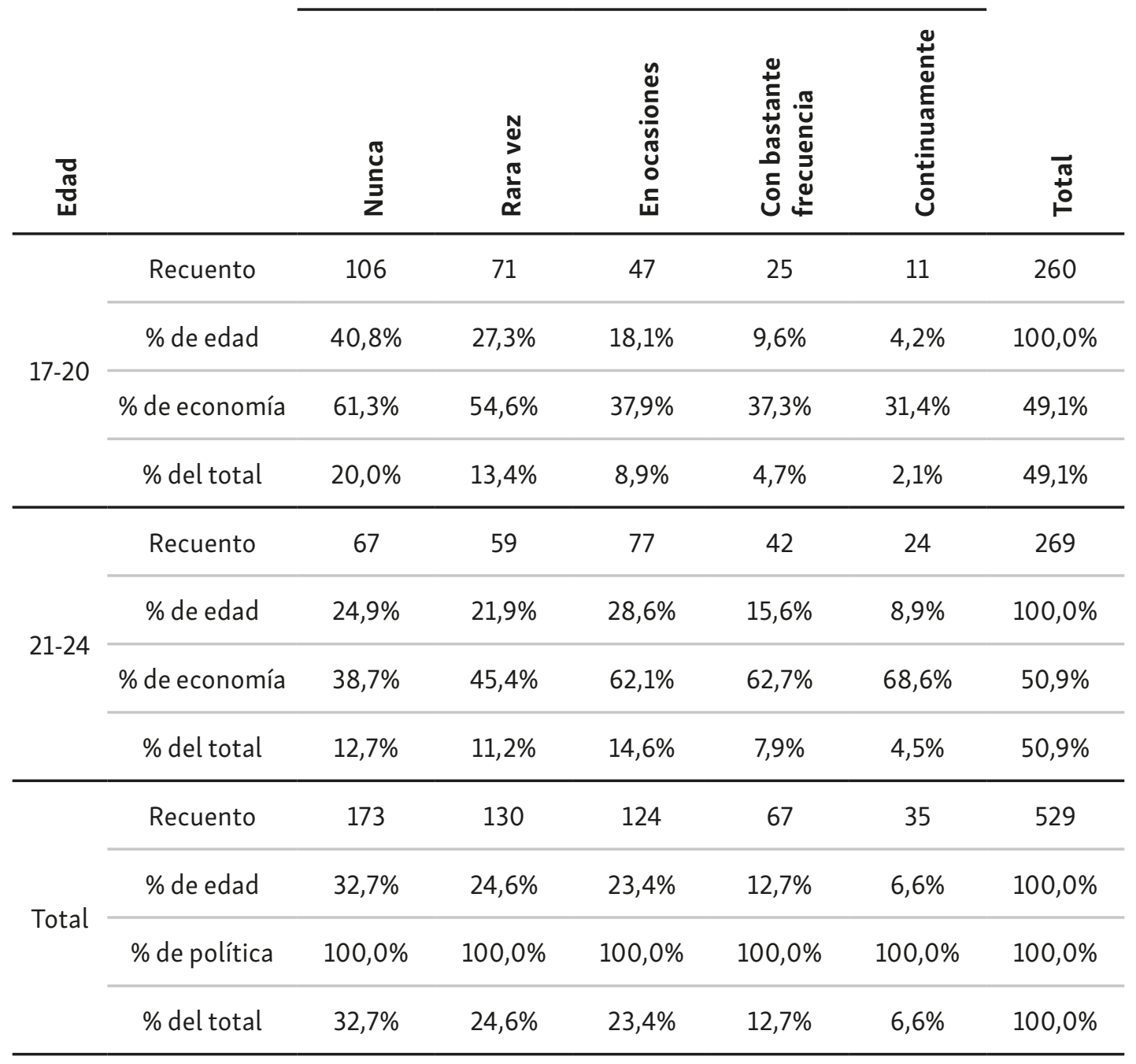

Tabla 5. Contingencia edad-consulta información económica

Fuente: Elaboración propia.

Todas las temáticas alcanzan una recurrencia superior en el grupo de 21 a 24 años debido, según sugieren los datos, a un mayor compromiso e interés por lo que ocurre en su entorno. La única excepción está marcada por noticias sobre famosos y celebrities. Englobadas mayoritariamente en la prensa rosa, son consultadas con bastante frecuencia o continuamente por 43,6\% de los de menor edad y entre $34,4 \%$ de 21 a 24 años. No se aprecian diferencias significativas en función del nivel educativo.

\section{Prácticas realizadas con las noticias}

Independientemente de su edad o nivel educativo, más de una cuarta parte $(26,5 \%)$ de los encuestados reconoce no contrastar la información porque confía en el primer medio al que accede y $17,5 \%$ no tiene tiempo para consultar otras fuentes. 


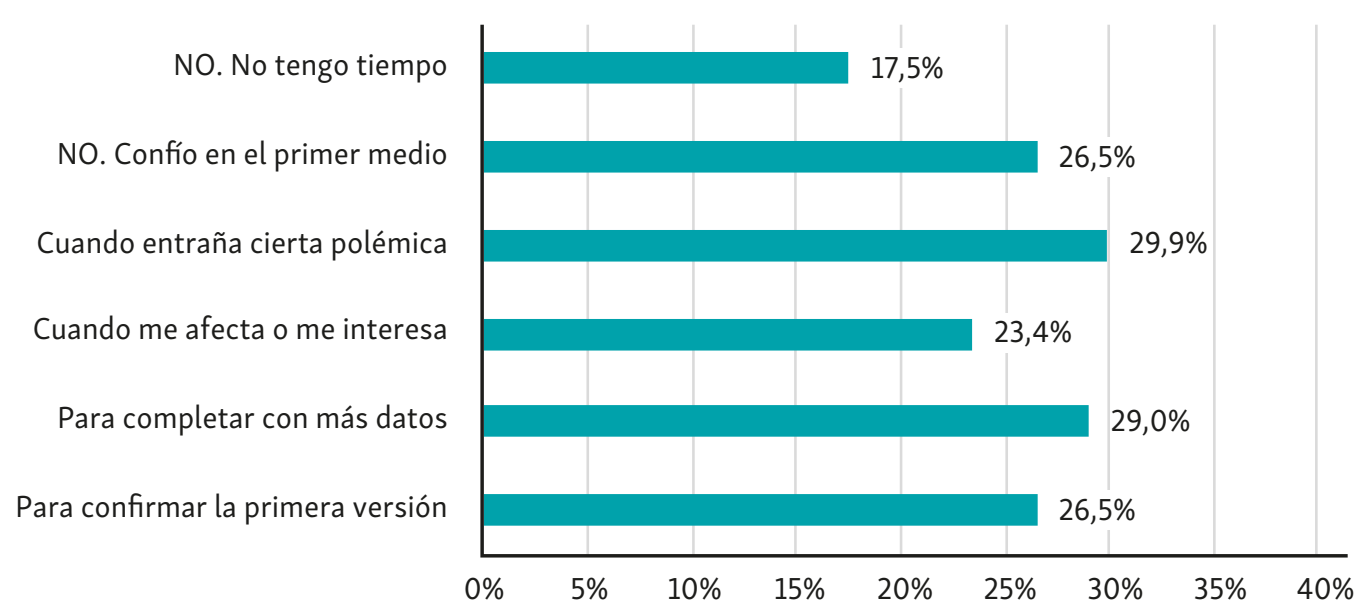

Figura 2. Contraste (o no) de la información y causas que le incitan a ello

Fuente: Elaboración propia.

Entre los que sí contrastan a veces, se observan porcentajes bastante equilibrados entre los que consultan otros medios cuando la información entraña cierta polémica $(29,9 \%)$ y cuando esta les afecta o interesa particularmente (23,4\%). Se presentan valores similares entre los que siempre las contrastan para confirmar la primera versión $(26,5 \%$ ) y los que pretenden completar con más datos (29\%) (figura 2). Tanto si realizan esta acción continuamente o en ocasiones, no se aprecian diferencias significativas en función de la edad o del nivel de estudios que cursan.

Los jóvenes prefieren comunicarse cara a cara para comentar las informaciones con su entorno. Entre uno (nunca) y cinco (continuamente), la media es de 3,75, valor que se aproxima a con bastante frecuencia. Esta alternativa es la única que aporta diferencias significativas en el nivel educativo (con mayor recurrencia entre los de estudios superiores). En torno a las herramientas asociadas a Internet, WhatsApp se acerca a la comunicación offline para compartir las informaciones $(\delta=3,64)$ y para comentarlas $(\delta=3,65)$. Incluso supera a las redes sociales, que alcanzan unas respectivas medias de 3,3 para compartir y de 3,32 para comentarlas. Raras veces emplean los blogs para comentar noticias $(\delta=2,46)$.

En cuanto a la edad, no se aprecian diferencias estadísticas en la comunicación cara a cara, pero sí en el entorno digital: los mayores comentan más las noticias tanto en redes como en WhatsApp y se inclinan más a compartirlas. Esta tendencia se rompe a la hora de difundirlas por las redes: entre 17 a 20 años lo realizan con una frecuencia superior $(\delta=3,4)$. Entre los de 21-24 años el valor es de 2,8.

\section{Confianza y percepción sobre las fuentes informativas}

Los jóvenes, en general, muestran bastante confianza hacia casi todas las vías de información, con una media que supera el tres sobre cinco, a excepción de los blogs, cuyo valor se sitúa en $\delta=2,84$. Estos resultados se relacionan en parte 
con el ya analizado escaso interés por contrastar las noticias del primer medio que consultan. Los valores más altos se registran en el formato analógico de los tradicionales: 3,33 para la prensa, 3,30 para la radio y 3,28 para la televisión. Este orden se rompe en las respectivas versiones digitales, aunque con menor confianza: una media de 3,14 para televisión en línea, 3,09 para la prensa y 3,06 para la radio. No obstante, este último dato debe ser interpretado con cautela, considerando que cerca de $10 \%$ contestó NS/NC, cuestión que también se relaciona con el escaso consumo de radio. En otras vías digitales menos convencionales, prefieren los buscadores como Google o Yahoo $(\delta=3,2)$ más que las redes sociales, cuyo índice es uno de los más bajos registrados $(\delta=3,09)$ (figura 3).

La edad no marca diferencias, pero sí el nivel educativo en dos de las versiones offline de los medios: $86,9 \%$ con niveles superiores muestra bastante, mucha o total confianza en la prensa en papel y $81 \%$ con menor educación, en la televisión.

Aunque no existe un referente claro entre los encuestados para calibrar la credibilidad de una información, la publicación de contenidos audiovisuales (imágenes fijas o videos) y la publicación en dos o más medios son los aspectos más valorados. Las respectivas medias alcanzadas son 1,81 y 1,80 sobre 5 . Estos valores se sitúan por encima de la autoría de la noticia $(\delta=1,78)$ y del reconocimiento cualitativo del medio $(\delta=1,75)$. Este último aspecto es el único que registra diferencias en función de la variable educación, con un sensible aumento entre los jóvenes que no tienen estudios superiores. El contenido de la información es lo menos valorado por todos los jóvenes $(1,66)$, sin divergencias significativas en función de los estudios. La edad tampoco se asocia a la credibilidad.

Independientemente de la edad y del nivel educativo, variables que no reflejan ninguna singularidad, el entretenimiento es la principal causa que motiva a los jóvenes a consultar la información de actualidad ( $\delta=2,6$ sobre 5). Frente a ello, pero en consonancia con los bajos valores registrados en la verificación de noticias, la causa menos recurrente es obtener diferentes perspectivas sobre lo que está pasando en su entorno $(\delta=2,2)$ (tabla 6$)$.

Finalmente, se confirma el bajo consumo deliberado frente al incidental. Los jóvenes no suelen informarse a pesar de que admiten que es importante $(\delta=2,6)$. Quienes sí lo hacen lo justifican porque les afecta directamente $(2,27)$ o porque les permite tomar alguna decisión en el ámbito político o económico $(2,17)$. Precisamente, estas áreas de interés periodístico ofrecen un bajo consumo. Tampoco en estos casos se registran diferencias en función de su nivel de estudios. En relación con la edad, se observa algo más de desacuerdo entre los de 21-24 años, dado que se decantan por la afirmación de que "es importante pero no lo suelo hacer". 


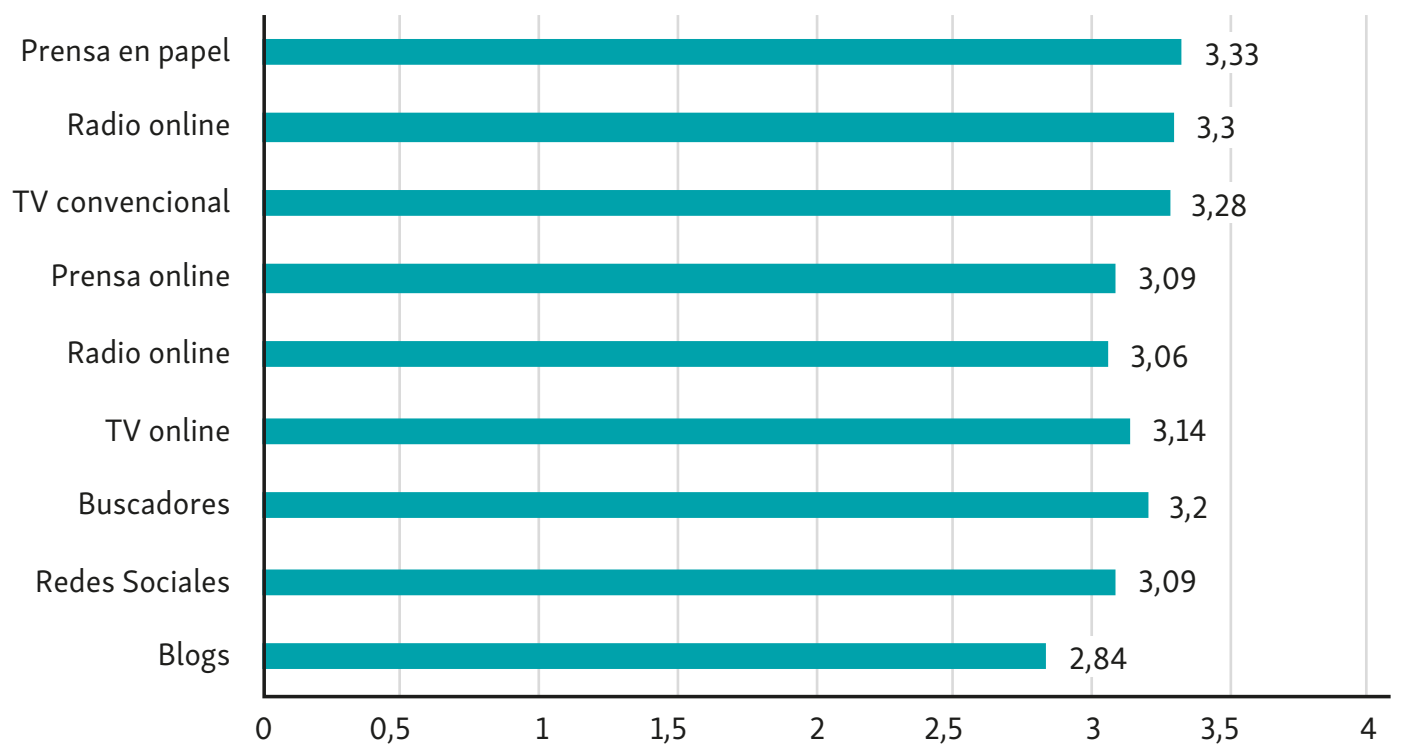

Figura 3. Media de confianza en las vías de acceso a las noticias

Fuente: Elaboración propia.

\begin{tabular}{cccccc} 
& Válidos & Perdidos & Media & Mediana & Moda \\
\hline $\begin{array}{c}\text { Porque es necesario implicarme con mi } \\
\text { entorno y participar como ciudadano }\end{array}$ & 528 & 5 & 2,23 & 2 & 2 \\
\hline $\begin{array}{c}\text { Para obtener diferentes perspectivas } \\
\text { de lo que está pasando }\end{array}$ & 530 & 3 & 2,2 & 2 & 2 \\
\hline Por mi trabajo o estudios & 527 & 6 & 2,4 & 2 & 2 \\
\hline $\begin{array}{c}\text { Para poder conversar con otras } \\
\text { personas }\end{array}$ & 528 & 5 & 2,28 & 2 & 2 \\
\hline \begin{tabular}{c} 
Solo la consulto porque me entretiene \\
\hline $\begin{array}{c}\text { Adquirí la costumbre en mi entorno } \\
\text { familiar }\end{array}$
\end{tabular} & 527 & 6 & 2,6 & 2 & 2 \\
\hline
\end{tabular}

Tabla 6: Causas para informarse. Escala de Likert 1- 5

Fuente: Elaboración propia.

\section{DISCUSIÓN Y CONCLUSIONES}

El primer hallazgo de este trabajo confirma que el consumo de noticias por parte de los jóvenes y adolescentes es bajo en medios tradicionales, tanto en prensa en papel como en medios audiovisuales o digitales. Como apunta la literatura sobre el particular (Bergström \& Jervelycke Belfrage, 2018; Mitchelstein \& Boczkowski, 2018; Mumtaz et al., 2019), consumen noticias fundamentalmente en plataformas de medios sociales o WhatsApp, a excepción del consumo de noticias en televisión 
dos o tres veces por semana. Este fenómeno podría estar relacionado con un hábito en el entorno familiar, supuesto no contemplado en este estudio y que puede abrir nuevas líneas de investigación. La falta de apego entre las generaciones más jóvenes hacia los medios periodísticos induce a pensar en su declive. Esto es un motivo de preocupación si se considera la cantidad de desinformación que circula en otras vías que recogen noticias de actualidad, asociada, entre otros fenómenos negativos, a las fake news y a los clickbaits, que no entrañan el deseable rigor periodístico. La adaptación digital de los medios de comunicación profesionales no supone una causa suficiente para atraer a estas audiencias, aunque también es cierto que en este trabajo no se confirma el trasvase a un consumo de videos informativos en otros canales y plataformas (Vara-Miguel, 2019).

Asimismo, los resultados están en la línea con Boczkowski y sus colegas (2017), que apuntan al consumo incidental como un elemento clave: los jóvenes madrileños consumen sobre todo noticias recibidas desde su entorno, a través de herramientas digitales o cara a cara, en mayor medida que las que buscan y consultan en los medios tradicionales. Este consumo incidental y el aparente conformismo que los jóvenes tienen por informarse mediante lo más accesible plantea otra reflexión: la inquietud, característica asociada a la juventud, no se refleja en buscar información de actualidad con rigor. A pesar de que el consumo de noticias se asocia con el compromiso social (Zhanh \& Shoric, 2018), no está entre sus prioridades.

Por otra parte, se confirma que la edad es un factor distintivo en el consumo. A mayor edad, se incrementa la búsqueda deliberada de noticias (Bergström \& Jervelycke Belfrage, 2018). Un mayor nivel de estudios también influye en más consumo de noticias. El tratamiento de esta variable representa uno de los aportes más importantes de este estudio. Futuras investigaciones podrían analizarla con mayor profusión y asociarla con mayor énfasis a la alfabetización mediática y digital. Sería interesante comprobar los aspectos que podrían incidir en la actitud de los jóvenes hacia un mayor interés por los medios profesionales periodísticos.

Desde una óptica temática, los asuntos que más interés despiertan en los encuestados son curiosidades y entretenimiento, tecnología, deportes y cultura. Entre los que menos, política y economía. Es relevante también que estas últimas, dos de las secciones con mayor protagonismo en los medios de comunicación generalistas, son las que menos les interesan. Ello podría vincularse al supuesto planteado en esta investigación sobre su notable desentendimiento por la mayoría de los medios eminentemente periodísticos.

En torno a las prácticas de los encuestados tras informarse, cabe destacar que una buena parte no verifica las noticias. Estos resultados preocupan, por la difusión masiva de la desinformación en el entorno digital que hace necesario contrastar 
y verificar las informaciones. Sorprendentemente, en esta franja de edad, a pesar de ser nativos digitales, suelen comentar las noticias en un entorno social offline. Son los jóvenes de más edad quienes más las comparten por vía digital. WhatsApp es la herramienta más utilizada, con un nivel superior al de los medios sociales.

Entre las causas por las que verifican la información destaca, en primer lugar, las noticias que entrañan cierto grado de polémica, seguida del interés por completar la noticia con más datos. Los que no las contrastan argumentan sobre todo que confían en el primer medio por el que reciben las noticias y, en menor medida, porque no tienen tiempo.

En cuanto al grado de confianza en la información, se puede decir que es alto, salvo para el blog. La prensa, la radio y la televisión, en formato analógico, ocupan, en ese orden, las preferencias de los encuestados, si bien la radio presenta un bajo consumo. Entre los medios digitales, destacan los buscadores por encima de las redes sociales, si bien este dato no se corresponde directamente con el consumo, que es mayor a través de las redes. Dos elementos principales actúan a favor de la credibilidad de las noticias: la presencia de documentos audiovisuales (videos o fotografías) y su publicación en varios medios. La variable edad vuelve a establecer un sesgo, pues los mayores contrastan más las noticias y tienen más oportunidad de comprobar que las informaciones son publicadas por varios medios.

Respecto del nivel educativo, no se aprecian variaciones en cuanto al nivel de confianza en los distintos tipos de medios y todos ellos, independientemente de su grado educativo, reconocen que es importante hacerlo, aunque no se informen lo suficiente.

Otro aspecto clave para entender el fenómeno del consumo de noticias por parte de los jóvenes es la relación con su compromiso cívico y ciudadano (Brites \& Kõuts-Klemm, 2018). En este punto, es habitual relacionar un acceso débil a las noticias con cierto grado de desafección política y social. Sin embargo, se puede aplicar un enfoque diferente. Por un lado, es patente la disposición de la juventud de incorporarse al mundo laboral y a la vida adulta, lo que se podría entender como manifestación de su implicación cívica y ciudadana. Por otro lado, la diversidad y el desplazamiento en lo que se refiere a los canales para acceder a las noticias y sus diferentes formatos probablemente conduce a pensar en un enfrentamiento al status quo tradicional, tanto político como mediático. Emplear nuevas experiencias de consumo de información de actualidad se podría ver, por lo tanto, como una forma de construcción del aprendizaje social en un nueva arena social y política.

La primera limitación del estudio es obtener los datos en un momento específico, que no hace posible analizar tendencias de consumo. Los resultados 
son parcialmente extrapolables a los jóvenes españoles, pues se centran en la Comunidad de Madrid. Otra limitación es establecer como únicas variables independientes la edad y el nivel educativo. Futuras investigaciones pueden tratar otras comparativas, relacionadas con el género o el estatus socioeconómico y su relación con la brecha digital. Estudiar el impacto de factores como las propias experiencias que podrían marcar el consumo de noticias y su interrelación con la propia conciencia ciudadana también sería un aporte.

Como principales contribuciones destaca la novedad en cuanto a los temas noticiosos preferidos por los jóvenes, a partir de una muestra representativa y resultados relevantes para descifrar el impacto de la edad y nivel de estudios en los patrones de consumo informativo. Finalmente, se observa la importancia de la comunicación cara a cara entre las acciones relacionadas con el consumo de noticias y las diferencias entre consumo y confianza en el medio de acceso a la noticia.

\section{FINANCIAMIENTO}

Proyecto nacional (España) de I+D+i "Redes sociales, adolescentes y jóvenes: convergencia de medios y cultura digital" (CSO2016-74980C2-2-R) Investigador Principal: Antonio García Jiménez. Fechas: 29/12/2016-29/06/2021.

\section{REFERENCIAS}

Aramburú-Moncada, L. G. \& López-Redondo, I. (2018). Pautas de consumo de noticias en las nuevas generaciones. Un análisis aplicado a estudiantes de periodismo de la Universidad de Sevilla (Media consumption patterns on new generations. Behavioral analysis applied to journalism students from the University of Seville). Ámbitos. Revista Internacional de Comunicación, 3(42), 76-96. https://doi.org/10.12795/Ambitos.2018.i42.05

Arceneaux, K. \& Johnson, M. (2013). Changing minds or changing channels? Partisan news in an age of choice. University of Chicago Press. https://doi.org/10.1002/polq.12243

Benedicto, J. (Dir.) (2017). Informe Juventud en España 2016. INJUVE. Retrieved from http://www.injuve.es/sites/default/files/2017/24/publicaciones/informe-juventud-2016.pdf

Bergström, A. \& Jervelycke Belfrage, M. (2018). News in social media: incidental consumption and the role of opinion leaders. Digital Journalism, 6(5), 583-598.

https://doi.org/10.1080/21670811.2018.1423625

Boczkowski, P., Mitchelstein, E., \& Matassi, M. (2017, January). Incidental news: How young people consume news on social media. In Proceedings of the 50th Hawaii International Conference on System Sciences (pp. 1785-1792). https://doi.org/10.24251/HICSS.2017.217 
Boczkowski, P. J., Mitchelstein, E., \& Matassi, M. (2018). “News comes across when I'm in a moment of leisure": Understanding the practices of incidental news consumption on social media. New Media \& Society, 20(10), 3523-3539. https://doi.org/10.1177/1461444817750396

Bode, L. (2016). Political news in the news feed: Learning politics from social media. Mass Communication \& Society, 19(1), 24-48. https://doi.org/10.1080/15205436.2015.1045149

Brites, M. J. \& Kõuts-Klemm, R. (2018). News definitions and motivations: youth and adults in Portugal and in Estonia. Estudos em Comunicação, 1(27), 175-190.

https://doi.org/10.20287/ec.n27.v1.a11

Casero-Ripollés, A. (2012). Más allá de los diarios: el consumo de noticias de los jóvenes en la era digital (Beyond newspapers: News consumption among young people in the digital era). Comunicar, 39, 151-158. https://doi.org/10.3916/C39-2012-03-05

Catalina-García, B., Sousa, J. P., \& Cristina Silva Sousa, L. C. (2019). Consumo de noticias y percepción de fake news entre estudiantes de Comunicación de Brasil, España y Portugal (Consumption of news and perception of fake news among Communication students from Brazil, Spain and Portugal). Revista de Comunicación, 18(2), 93-115. https://doi.org/10.26441/RC18.2-2019-A5

Catalina-García, B., García-Jiménez, A., \& Montes-Vozmediano, M. (2015). Jóvenes y consumo de noticias a través de Internet (Youths and consumption of news via Internet and social media). Historia y Comunicación Social, 20(2), 601-619.

https://doi.org/10.5209/rev_HICS.2015.v20.n2.51402

Cherian, J. \& Jacob, J. (2012). Analysis of Attitude towards Online and Print Publications: A Case Study among University Students. Asian Social Sciences, 9(1), 52-59. https://doi.org/10.5539/ass.v9n1p52

Condeza-Dall'Orso, R., Bachmann-Cáceres, I., \& Mujica-Holley, C. (2014). El consumo de noticias de los adolescentes chilenos: intereses, motivaciones y percepciones sobre la agenda informativa (News consumption among Chilean adolescents: Interest, motivations and perceptions on the news agenda). Comunicar, 43, 55-64. https://doi.org//10.3916/C43-2014-05

Costera, I. (2007). The paradox of popularity. How young people experience news. Journalism Studies, 8(1), 96-116. https://doi.org/10.1080/14616700601056874

Drok, N., Hermans, L., \& Kats, K. (2018). Decoding youth DNA: The relationship between social engagement and news interest, news media use and news preferences of Dutch millennials. Journalism, 19(5), 699-717. https://doi.org/10.1177/1464884917703469

Edgerly, S., Vraga, E. K., Bode, L., Thorson, K., \& Thorson, E. (2018a). New media, new relationship to participation? A closer look at youth news repertoires and political participation. Journalism \& Mass Communication Quarterly, 95(1), 192-212. https://doi.org/10.1177/1077699017706928

Edgerly, S., Thorson, K., Thorson, E., Vraga, E. K., \& Bode, L. (2018b). Do parents still model news consumption? Socializing news use among adolescents in a multi-device world. New Media \& Society, 20(4), 1263-1281. https://doi.org/10.1177/1461444816688451

Fernández de Arroyabe-Olaortua, A., M., Lazkano-Arrillaga, I., \& Eguskiza-Sesumaga, L. (2018). Nativos digitales: Consumo, creación y difusión de contenidos audiovisuales online (Digital natives: Online audiovisual content consumption, creation and dissemination). Comunicar, 57, 61-69. ttps://doi.org/10.3916/C57-2018-06 
Flanagin, A. J. \& Metzger, M. J. (2008). Digital Media and Youth: Unparalleled Opportunity and Unprecedented Responsibility. In M. J. Metzger y A. J. Flanagin (Eds.), Digital Media, Youth, and Credibility (pp. 5-27). Cambridge, MA: MIT Press. https://doi.org/10.1162/dmal.9780262562324.005

Fletcher, R. \& Nielsen, R. K. (2018). Are people incidentally exposed to news on social media? A comparative analysis. New media \& society, 20 (7), 2450-2468. https://doi.org/10.1177/1461444817724170

Gangadharbatla, H., Bright, L. F., \& Logan, K. (2014). Social Media and news gathering: tapping into the millennial mindset. The Journal of Social Media in Society, 3(1), 45-63. Retrieved from http://thejsms.org/index.php/TSMRI/article/view/63/33

Hasebrink, U. \& Popp, J. (2006). Media repertoires as a result of selective media use. A conceptual approach to the analysis of patterns of exposure. Communications, 31(3), 369-387. https://doi.org/10.1515/COMMUN.2006.023

Hermida, A., Fletcher, F., Korell, D., \& Logan, D. (2012). Share, like, recommend. Decoding the social media news consumer. Journalism Studies, 13(5-6), 815-824. https://doi.org/10.1080/1461670X.2012.664430

Hernández-Serrano, M. J., Renés-Arellano, P., Graham, G., \& Greenhill, A. (2017). From prosumer to prodesigner: participatory news consumption. Comunicar, 50, 77-88. https://doi.org/10.3916/C50-2017-07

Hilligoss, B. \& Rieh, S. Y. (2007). Developing a unifying framework of credibility assessment: construct, heuristics, and interaction in context. Information Processing and Management, 44(4), 1467-1484. https://doi.org/10.1016/j.ipm.2007.10.001

Injuve. (2014). Sondeo de opinión: jóvenes, ocio y consumo (Opinion poll: youth,leisure and consumption). Retrieved from http://www.injuve.es/sites/default/files/conclusionessondeo_2014-3.pdf

Johnson, T. J. \& Kaye B. K. (2014). Credibility of social network sites for political information among politically interested internet users. Journal of Computer-Mediated Communication, 19(4), 957-974. https://doi.org/10.1111/jcc4.12084

Kang, Y. C. (2009). Communication technologies: Diffusion of online news use and credibility among young web users in the information age (Doctoral dissertation, University of Nevada, Las Vegas). Retrieved from https://digitalscholarship.unlv.edu/cgi/viewcontent. cgi?article $=1160 \&$ context $=$ thesesdissertations

Kaufhold, K. (2010). Journalists show unified optimism about young adults' news consumption. Newspaper Research Journal, 31(2), 63-68. https://doi.org/10.1177/073953291003100206

Kim, K. S., Sin, S. C. J., \& Yoo-Lee, E. (2014). Undergraduates' use of social media as information sources. College \& Research Libraries, 75(4), 442-457. https://doi.org/10.5860/crl.75.4.442

Kim, Y., Chen, H. T., \& Gil de Zúñiga, H. (2013). Stumbling upon news on the internet: Effects of incidental news exposure and relative entertainment use on political engagement. Computers in Human Behavior, 29(6), 2607-2614. https://doi.org/10.1016/j.chb.2013.06.005

Lee, H. \& Yang, J. (2014). Political knowledge gaps among news consumers with different news media repertoires across multiple platforms. International Journal of Communication, 8, 597-617. Retrieved from https://ijoc.org/index.php/ijoc/article/view/2455 
Martínez Costa, M. P., Serrano Puche, J., Portilla, I., \& Sánchez Blanco, C. (2019). Young adult's interaction with online news and advertising. Comunicar, 59, 19-28. https://doi.org/10.3916/C59-2019-02

Matsa, K. E., Silver, L., Shearer, E., \& Walker, M. (2018, October 30). Western Europeans under 30 view news media less positively, rely more on digital platforms than older adults. Pew Research Center. Retrieved from hhttps://www.journalism.org/2018/10/30/ western-europeans-under-30-view-news-media-less-positively-rely-more-on-digitalplatforms-than-older-adults/

Metzger, J. M., Flanagin, A., \& Zwarun, L. (2003). College students' web use, perceptions of information credibility, and verification behavior. Computers \& Education, 41(3), 271-290. https://doi.org/10.1016/S0360-1315(03)00049-6

Mitchelstein, E. \& Boczkowski, P. J. (2018). Juventud, estatus y conexiones. Explicación del consumo incidental de noticias en redes sociales (Youth, Status and Connections. Explanation of the Incidental Consumption of News in Social Networks). Revista Mexicana de Opinión Pública, (24), 131-145.https://doi.org/10.22201/fcpys.24484911e.2018.24.61647

Mumtaz, T., Karamat, K., \& Iqbal, A. (2019). A comparative study of traditional and social media consumption patterns among youth. Journal of Media Studies, 30(2), 155-170. http://111.68.103.26/journals/index.php/jms/article/viewFile/2011/780

Negredo, S., Amoedo, A., Vara-Miguel, A., Moreno, E., \& Kaufmann, J. (2021). Digital New Report.es. Retrieved fromhttps://www.digitalnewsreport.es/resumen-ejecutivodigitalnewsreport-es-2021-periodismo-de-calidad-y-cercania-para-combatir-la-infodemia/

Newman, N., Fletcher, R., Schulz, N., Andi, S., \& Nielsen, R. S. (2020). Reuters Institute Digital News Report 2020. Retrieved from https://reutersinstitute.politics.ox.ac.uk/sites/ default/files/2020-06/DNR_2020_FINAL.pdf

Papathanassopoulos, S., Coen, S., Curran, J., Aalberg, T., Rowe, D., Jones, P., \& Tiffen, R. (2013). Online threat, but television is still dominant: A comparative study of 11 nations' news consumption. Journalism Practice, 7(6), 690-704.https://doi.org/10.1080/17512786.2012.761324

Patterson, T. E. (2007). Young People and News. A Report from the Joan Shorenstein Center on the Press, Politics and Public Policy. Harvard University, John F. Kennedy School of Government. Retrieved frohttps://shorensteincenter.org/wp-content/uploads/2012/03/ young_people_and_news_2007.pdf

Qayyum, M. A., Williamson, K., Liu, Y. H., \& Hider, P. (2010). Investigating the news seeking behavior of young adults. Australian academic \& Research libraries, 41(3), 178-191.https:// doi.org/10.1080/00048623.2010.10721462

Soengas-Pérez, X., López-Cepeda, A. M., \& Sixto-García, J. (2019). Dieta mediática, hábitos de consumo de noticias y desinformación en los universitarios españoles (Media diet, consumption habits and misinformation among Spanish university students). Revista Latina de Comunicación Social, (74), 1056-1070http://www.revistalatinacs.org/074paper/1371/54es.html

Swart, J., Peters, C., \& Broersma, M. (2019). Sharing and Discussing News in Private Social Media Groups: The social function of news and current affairs in location-based, workoriented and leisure-focused communities. Digital Journalism, 7(2), 187-205.https://doi.or $\underline{\mathrm{g} / 10.1080 / 21670811.2018 .1465351}$ 
Vara-Miguel, A., Negredo, S., Amoedo, A., \& Moreno, E. (2019). Digitalnewsreport.es 2019. Los usuarios españoles se previenen de la desinformación y demandan a los medios mayor profundidad y vigilancia (Spanish users in guard against misinformation and demand greater depth and vigilance from the media). Digital News Report. Retrieved fromhttp://www.digitalnewsreport.es/los-usuarios-espanoles-se-previenen-de-ladesinformacion-y-demandan-a-los-medios-mayor-profundidad-y-vigilancia/

Wackowski, O. A., Sontag, J. M., \& Hammond, D. (2019). Youth and young adult exposure to and perceptions of news media coverage about e-cigarettes in the United States, Canada and England. Preventive medicine, (121), 7-10.http://doi.org/10.1016/j.ypmed.2019.01.013

Wonneberger, A. \& Kim, S. J. (2017). TV News Exposure of Young People in Changing Viewing Environments: A Longitudinal, Cross-National Comparison Using People-Meter Data. International Journal of Communication, (11), 72-93. Retrieved https://ijoc.org/index.php/ijoc/article/view/5384

Yuste, B. (2015). Las nuevas formas de consumir información de los jóvenes (New ways of consuming information from young people). Revista de Estudios de Juventud, (108), 179-191. Retrieved fromhttp://www.injuve.es/sites/default/files/2017/46/publicaciones/ revista108_14-nuevas-formas-consumir-informacion.pdf

Zhang, N. \& Skoric, M. M. (2018). Media use and environmental engagement: Examining differential gains from news media and social media. International Journal of Communication, (12), 24. Retrieved from https://ijoc.org/index.php/ijoc/article/view/7650

\section{SOBRE LOS AUTORES}

BEATRIZ CATALINA-GARCía, Profesora contratada doctora en la Universidad rey Juan Carlos. Doctora en Ciencias de la Comunicación (URJC). Licenciada en Periodismo (Universidad Complutense de Madrid) y Licenciada en Ciencias Políticas (UNED). Sus líneas de investigación se centran en Audiencias, Menores y Jóvenes en Internet y Comunicación pública digital. Entre sus últimos trabajos destaca "The dilemmas of parental mediation: Continuities from parenting in general" (2020).

(iD) https://orcid.org/0000-0003-0464-3225

ANTONIO GARCíA-JIMÉNEZ, Doctor en Ciencias de la Información, es catedrático de Periodismo en la Universidad Rey Juan Carlos de Madrid. Ha sido Decano de la Facultad de Ciencias de la Comunicación en esta universidad (2008-2014). Su investigación se centra en los menores y jóvenes en Internet y en la documentación y los datos en los medios. Uno de sus últimos trabajos es "Investigación del comportamiento de menores y jóvenes en las redes sociales mediante técnicas de Social Big Data” (2021).

https://orcid.org/0000-0002-8423-9486

PEDRo PANIAGUA-SANTAMARÍA, Profesor titular de periodismo en la Universidad Complutense de Madrid. Es autor de Líneas al margen (Verbum, 2019), De Midas a Goldman Sachs. La estela de la codicia (Amazon, 2013), Breve historia del futuro (Taurus, 2012), Los géneros en la Red: reportaje, entrevista y crónica (Fragua, 2015), Cultura y guerra del fútbol (UOC, 2009), Información e interpretación en periodismo (UOC, 2009), Información deportiva (Fragua, 2003) y La prensa ante la televisión privada (UCM, 2001). 